\title{
NUMERICAL MODELLING OF CONCENTRATION PROFILES IN MEMBRANE CHANNEL
}

\section{Ali K. Abdel-Rahman}

Associate Professor, Faculty of Engineering, Assiut University, Assiut, Egypt

\author{
Abdul Aziz Abbara \\ Department of Food Engineering, Al-Baath University, Homs, Syria \\ Mohamed R. Bayoumi \\ Professor, Faculty of Engineering, Assiut University, Assiut, Egypt
}

(Received December 10, 2005 Accepted December 31, 2005)

\begin{abstract}
Numerical modelling of the flow and concentration polarization in pressure driven membrane process is challenged by the complex coupling in the flow, mass and energy equations along with any added effects of variable solution properties. The present study is directed towards the establishment of a numerical method capable of predicting the flow and concentration characteristics of the laminar channel flows with permeable wall taking into account the variable properties of the stream. The present results highlights the necessity of the fine mesh grid near the membrane wall to cope with the very steep changes in concentration and temperature there and to the importance of making the permeation velocity vary with concentration. The model was validated and the results showed a good agreement with the previous numerical and experimental results.
\end{abstract}

KEYWORDS: Membrane, Numerical modelling, Concentration Polarization, Microfiltration, Ultrafiltration, Sugar solution.

\section{NOMENCLATURE}

\section{Symbols}

c concentration $\left(\mathrm{kg} / \mathrm{m}^{3}\right)$

$c_{w} \quad$ wall concentration $\left(\mathrm{kg} / \mathrm{m}^{3}\right)$

$c_{p} \quad$ specific heat capacity $(\mathrm{kJ} / \mathrm{kg} \mathrm{K})$

$D \quad$ Diffusion coefficient $\left(\mathrm{m}^{2} / \mathrm{sec}\right)$

$f \quad$ rejection of the membrane

$h \quad$ channel half-width (m)

$k_{t} \quad$ thermal conductivity $(\mathrm{W} / \mathrm{m} \mathrm{K})$

$P$ pressure $(\mathrm{Pa})$

$P e \quad$ Péclet number, $=h v_{w o} / D$

$R e_{o} \quad$ inlet Reynolds number $=4 h u_{m o} d v$

$R e_{w} \quad$ wall Reynolds number $=h v_{w o} / v$

$S_{\Phi} \quad$ source term of the variable $\phi$
$S_{p} \quad$ coefficient in the discretised source term

$S_{u} \quad$ coefficient in the discretised source term

Sc $\quad$ Schmidt number $=v / D$

$T$ temperature $\left({ }^{\circ} \mathrm{C}\right)$

$u$ mean streamwise velocity $(\mathrm{m} / \mathrm{s})$

$u_{m o}$ inlet cross-sectionally averaged streamwise velocity $(\mathrm{m} / \mathrm{s})$

$v \quad$ velocity in $y$ direction $(\mathrm{m} / \mathrm{s})$

$v_{w} \quad$ wall velocity $(\mathrm{m} / \mathrm{s})$ 


$v_{w o} \quad \begin{aligned} & \text { wall velocity at the beginning of } \\ & \text { permeation }(\mathrm{m} / \mathrm{s})\end{aligned}$
$x \quad \begin{aligned} & \text { Cartesian coordinate in the } \\ & \text { streamwise direction }\end{aligned}$
$y \quad \begin{aligned} & \text { Cartesian coordinate normal to } \\ & \text { the wall }\end{aligned}$

\section{Greek Symbols}

$\alpha_{D} \quad$ surface characteristics of membrane

$\beta \quad$ constant in Eq. (8)

$\delta \quad$ thickness of the concentration polarization layer $(\mathrm{m})$

$\phi \quad$ any of variables to be solved $\phi_{s} \quad$ slip coefficient

$\Gamma_{\Phi} \quad$ diffusion coefficient of the variable $\phi$

$\mu \quad$ dynamic viscosity (Pa. $\mathrm{s})$

$v \quad$ kinematic viscosity $=\mu / \rho\left(\mathrm{m}^{2} / \mathrm{s}\right)$

$\rho \quad$ density $\left(\mathrm{kg} / \mathrm{m}^{3}\right)$

$\xi \quad$ water recovery factor, $=v_{w o} x / h u_{m o}$

\section{Subscript}

$o \quad$ inlet

w wall

\section{INTRODUCTION}

Over the last years, membrane separation processes have been widely adopted by different industries. Today most membrane filtration systems use cross-flow (or tangential flow) filtration, where the pressure-driven feed stream flows across the membrane rather than through it. The solution passing through the membrane, containing solvents and solutes not retained by the membrane is called permeate while the solution containing the retained (rejected) species called retentate, reject stream, or concentrate. They are very attractive due to the large separation factors; low operating costs, absence of phase change and isothermal mode of operation [1].

Nevertheless, the performance of membrane separation process is severely affected by transient build up of a concentration polarization (CP) layer of rejected species at the membrane upstream interface [1,2]. As particle accumulation continues, particle concentration near the membrane surface reaches its maximum value and a particle cake layer or gel layer forms between the membrane surface and the CP layer. Further transport of particles to the membrane surface results in the growth of the cake layer until a steady state is attained. At steady state, the convective flux of particles carried toward the membrane with permeate is balanced by back-diffusion away from the membrane [3]. Particle accumulation in the cake layer and in the CP layer above the cake provides an additional resistance to permeate flow and, hence, reduces permeate flux [3].

Prediction of concentration profiles of particles at the concentration polarization layer and its effect on permeate flux and solute rejection is a necessary step in the design of an efficient system in the membrane filtration [4]. Different theoretical models such as the film model, the gel polarization model, the osmotic pressure model, and the resistance in series model have been widely used to predict concentration polarization and its effects on permeate flux and solute rejection [5].

However, the assumption that axial solute transport can be ignored, which is the basis for the film model, brings about a rather large internal scattering of the calculated values of the solute wall concentration, $c_{w}[6]$. Another effect of neglecting the axial 
transport of the solution is that the flux is averaged over the whole membrane length. Therefore, it is impossible to use the classical model to predict the influence of membrane length on the permeate flux [5].

Furthermore, empirical mass transfer correlations used in combination with these models are only adequate for low mass transfer fluxes and impermeable walls. However, these assumptions are not valid in systems with high permeation rates and in the presence of mixing promoters that induce recirculation zones in the flow [7].

Computational fluid dynamics (CFD), together with mass transfer modelling, has been proved to be a powerful tool to be used in the feed-side of membrane modules to effect the predictions of velocities, pressure and solute concentration, variables that are crucial for the management of the concentration polarisation phenomenon $[7,8,9]$. This allows for the prediction of the concentration polarisation without resorting to simplifying approaches such as the film theory.

Elimelech and Bhattacharjee [10] have compared the results of the combined film model and the osmotic pressure model with the results for the numerical solution from the two-dimensional convective- diffusion equation, which applies osmotic pressure model for the boundary condition flux. They found that numerical integration slightly overestimates the flux, while the analytical equation underestimates largely the flux. This is referred to the existence of a small error in the local flux estimation, which causes large error in the averaged flux. They pointed out that although constant values of the solute diffusivity were used in the model, incorporation of concentration dependent diffusivities would not significantly complicate the model.

Huang and Morrissey [11] used the finite-element analysis (FEA) for solving the concentration field given an approximate solution to the velocity field, by neglecting the diffusion in the $\mathrm{x}$-direction and assuming constant properties. This work highlighted the need for extremely high meshes near the vicinity of the membrane surface and relaxed towards the channel centerline because of the extremely thin concentration boundary layer.

Lee and Clark [12] performed a similar analysis to Huang and Morrissey [11] but this time solved the concentration equation using a finite difference method. They also included a shear induced diffusion term, as well as the usual molecular diffusion term, in the concentration equation. In addition, they developed an expression for the permeate flux which is based on diffusion through a stagnant cake layer assumed to develop on the membrane surface. Both works are limited by the fact that they assume the velocity field to be known.

Geraldes et al. [7] performed finite volume simulations for predicting the concentration polarisation for different nanofiltration (NF)/reverse osmosis (RO) operating conditions through a membrane for a simple channel flow. They solved both the flow and the concentration fields for a constant property situation. They concluded that the hybrid scheme appeared as the naturally elected scheme to discretise the transport equations in this type of flows. In later work, Geraldes et al. [13], have extended this model to include variable fluid properties. Their studies showed that higher main flow Reynolds numbers led to a smaller concentration polarisation. Additionally, for a given main flow Reynolds number, higher values of both the wall 
Reynolds number and Schmidt number induced an increase of the concentration polarisation.

Paris et al. [5] have solved the transport equation coupled with the resistance in series model by means of finite volume analysis, using a nonuniform mesh. The method was applied to ultrafiltration of dextran T500 solution using a tubular membrane. Their results showed that both the local thickness of concentration boundary layer and the local permeate flux at the membrane surface varied over the whole membrane length. On the other hand, the average concentration in boundary layer became quickly constant along the membrane. Thus, they concluded that the decrease of permeate flux along the membrane length was mainly due to an increase of boundary layer thickness rather than an increase of local concentration.

Wiley and Fletcher [8] have developed a general purpose finite volume model of concentration polarisation and fluid flow in pressure driven membrane separation processes. They have compared their results with its counterpart obtained using different models dealing with concentration polarisation and show that their model perform correctly from a quantitative and qualitative perspective. The validation process has highlighted the need for very fine meshes near the wall and the use of suitable high order numerical schemes, especially when the polarisation is high.

Damak et al. [14] described a method to couple the Navier-Stokes and Darcy equations using a finite difference scheme method for an isothermal, steady state, and laminar flow in a tubular domain with a permeable wall. In later work, Damak et al. [15] have extended their model to solve the convective diffusion equation for particle transport at the steady state for the case of nonuniform permeation velocity in laminar flow in a tubular channel with a cylindrical coordinate system. The equation was solved using a finite difference scheme. Results showed that wall Reynolds number was a very important parameter that controls particle deposition onto a permeable surface of membranes which influences the solute concentration profile and also controls the concentration polarisation along the tubular membrane.

The permeate flux values calculated in many studies are smaller than the experimental values. One of the reasons for the underestimation is the negligibility of the slip velocity effect on the mass transfer coefficient. In this sense, slip velocity means the axial velocity component on the membrane wall. Singh and Laurence [16] have studied in detail the influence of slip-velocity at a porous surface for a parallel flat membrane system assuming fully developed flow. They examined the effect of slip coefficient on velocity profiles, pressure gradient and concentration polarization in ultrafiltration. The equations of motion were solved by the regular perturbation method while the coupled diffusion equation in the boundary layer was solved using a finite difference scheme. Results showed that the velocity profiles flatten and approached a plug flow as the slip coefficient increased due to a decrease in the wall shear rate. Slip velocity increased with slip coefficient and approached an asymptotic value. Concentration polarization at the membrane surface was reduced as slip coefficient increased and the effect was more significant for low values of the normalized diffusion coefficient. It was observed that the effect of slip coefficient on polarization was to promote diffusive transport of solute molecules from the membrane surface to 
the bulk solution. The net effect of this was to reduce polarization and increase flux rates.

This is consistent with the finding of Yeh and Cheng [17] in which the concentration polarization decreases as the slip coefficient is increased. Yeh and Cheng [17] in their model also take into consideration concentration dependence on diffusivity and viscosity. The experimental flux data of ultrafiltration of Dextran T500 was used to verify the calculated results. The results showed that the slip flow of a macromolecular solution on the porous surface did exist and the permeate flow increased with the increase in the slip flow.

One or more of the following simplifications had been made in the previous studies: (a) the wall permeation velocity was assumed piece-wise or constant along the axial length; (b) the fluid flow field was approximated by some prescribed functions or by a reduced form of the momentum equation; (c) the wall velocity may depend on osmotic pressure but axial pressure drop was neglected or an approximate pressure drop was used without solving the momentum equation; and (d) the fluid transport properties were assumed constant or concentration-dependent viscosity and diffusion coefficient were used. None of the previous studies has realized the sensitivity of the sugar solution properties to the changes in both temperature and concentration. Thus, the present study is directed towards removing some of the assumptions discussed above and to establish a numerical method capable of predicting the flow and concentration characteristics of the laminar channel flows with permeable wall taking into account the variable properties of the stream. For this purpose, a finite volume discrete scheme using the SIMPLE (Semi-Implicit Method for Pressure Linked Equations) pressure-correction scheme combined with QUICK (Quadratic Upwind Interpolation Convective Kinematics) scheme in the frame of staggered grid is used. The simulation was carried out by an IBM compatible personal computer with 450 MHz Pentium III processor, 64 MB RAM memory and Windows 98 operating system.

\section{MODEL PROBLEM AND DISCRETIZATION}

\subsection{Governing Equations}

In crossflow membrane filtration the feed stream, which flows mainly tangentially to the porous membrane surface, is modeled by the Navier-Stokes, mass and energy transfer equations by the two dimensional convective and diffusion equation.

\subsubsection{Flow Model}

For two dimensional, incompressible, steady laminar channel flows; the continuity and Navier-Stokes equations are given as [18];

$$
\begin{aligned}
& \frac{\partial u}{\partial x}+\frac{\partial v}{\partial y}=0 \\
& \frac{\partial(\rho u u)}{\partial x}+\frac{\partial(\rho v u)}{\partial y}-\frac{\partial}{\partial x}\left[\mu\left(\frac{\partial u}{\partial x}\right)\right]-\frac{\partial}{\partial y}\left[\mu\left(\frac{\partial u}{\partial y}\right)\right]+\frac{\partial P}{\partial x}=0
\end{aligned}
$$




$$
\frac{\partial(\rho u v)}{\partial x}+\frac{\partial(\rho v v)}{\partial y}-\frac{\partial}{\partial x}\left[\mu\left(\frac{\partial v}{\partial x}\right)\right]-\frac{\partial}{\partial y}\left[\mu\left(\frac{\partial v}{\partial y}\right)\right]+\frac{\partial P}{\partial y}=0
$$

Where, $u$ and $v$ are the streamwise and transverse velocity components, respectively.

\subsubsection{Mass Transfer Model}

Mass transfer occurring within domains with porous walls can be mathematically expressed by the two dimensional convective and diffusion equation as follows $[8,19]$;

$$
\frac{\partial(\rho u c)}{\partial x}+\frac{\partial(\rho v c)}{\partial y}-\frac{\partial}{\partial x}\left[D\left(\frac{\partial c}{\partial x}\right)\right]-\frac{\partial}{\partial y}\left[D\left(\frac{\partial c}{\partial y}\right)\right]=0
$$

Where $D$ is the solute diffusion coefficient and $c$ is the solute concentration. The solute transport introduces the Schmidt number, $S c$, and the Péclet number, $P e$. The Schmidt number and the Péclet number are defined as;

$$
S c=v / D \text {, and } \quad P e=h v_{w o} / D
$$

where $v_{w o}$ is the initial wall permeation velocity, $h$ is the channel half width and $v$ is the fluid kinematic viscosity.

\subsubsection{Energy transfer model}

For two dimensional, incompressible, steady laminar channel flows; the energy equation is given as [18]

$$
\frac{\partial(\rho u T)}{\partial x}+\frac{\partial(\rho v T)}{\partial y}-\frac{\partial}{\partial x}\left[\frac{k_{t}}{c_{p}}\left(\frac{\partial T}{\partial x}\right)\right]-\frac{\partial}{\partial y}\left[\frac{k_{t}}{c_{p}}\left(\frac{\partial T}{\partial y}\right)\right]=0
$$

where $k_{t}$ and $c_{p}$ are the fluid thermal conductivity and specific heat at constant pressure, respectively.

\subsection{Computational Domain and Grid System}

The physical problem considered in the present study is steady state laminar channel flow with permeable walls. Figure 1 displays the flow geometry and the coordinate system used for the problem considered. There are two dynamic nondimensional parameters, the main flow Reynolds number at the inlet to the computational domain and the wall Reynolds number. They are denoted by $R e_{o}$ and $R e_{w}$, respectively and defined as [20];

$$
R e_{o}=\frac{4 h u_{m o}}{v} \quad \text {, and } \quad R e_{w}=\frac{h v_{w o}}{v}
$$

where $u_{m o}$ is the cross-sectionally averaged streamwise velocity at the inlet to the computational domain. The local permeation velocity through the concentration polarization and membrane $v_{w}$ was first set to a constant value equal to that at the beginning of permeation $v_{w o}$. In other cases, the permeation velocity was specified as a 
function of the wall concentration of solute. This leads to a more physically meaningful solution.

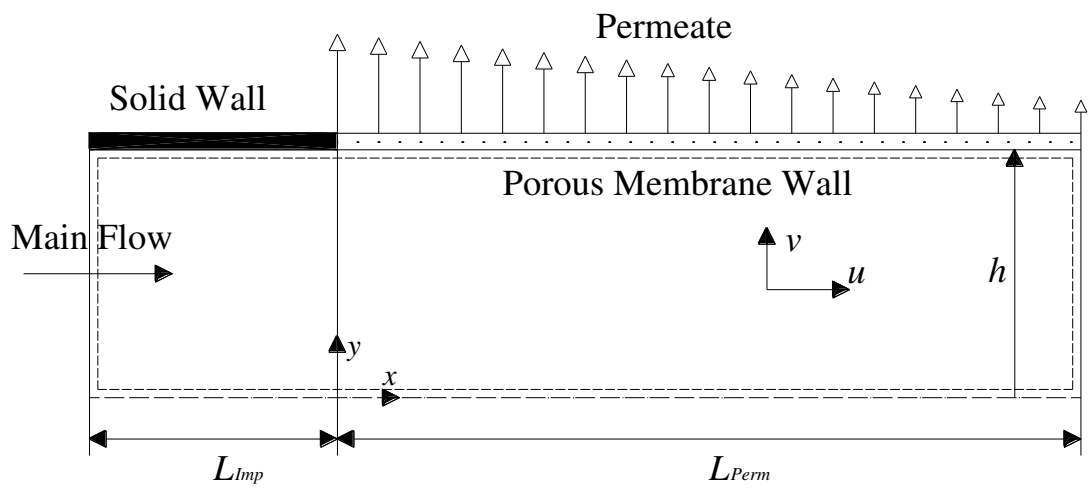

Figure 1: Schematic diagram of flow geometry and computational domain.

The computations are carried out in the rectangular domain shown in Fig. 1. The origin of the streamwise coordinate, $x$, is located at the beginning of the membrane and the origin of the normal coordinate, $y$, is set at the horizontal midplane of the channel. The position of the inlet section for the computational domain is located at a position $L_{\text {Imp }}$ upstream from the starting point of the membrane, and that of the outlet section is located at a position $L_{P e r m}$ downstream from the starting point of the membrane. The main result were obtained for a computational domain of $-10 \leq x / h \leq 100$, i.e. $L_{\text {Imp }}=10$ $h$ and $L_{\text {Perm }}=100 h$.

One of the important points of numerical solution is the choice of grid point system for which the equations are discretised. In relation with the use of the SIMPLE scheme, a staggered grid is used in this work [21]. A nonuniform grid system was employed and the grid density was made higher near the leading edge of the membrane in $x$ direction and near the walls in the $y$ direction to take into accounts the steep change of velocity, temperature and concentration there. Grid points of total number of $251 \times 100$ or $251 \times 200$ were allocated in the computational domain depending on the solute diffusion coefficient value. The smaller the solute diffusion coefficient the finer the grid points are required. In each of the two cases about 70 percent of the crossstream grids were located at a distance of $0.2 \mathrm{~h}$ from the membrane wall (the maximum step size in the $y$-direction needs to be less than or equal to $1 \times 10^{-5}$ ). This is proved to be an important requirement for the stability of numerical procedure.

\subsection{Discretization}

The steady-state form of the conservation equations of continuity, momentum, energy and concentration can be written in a general form as [7,13,18];

$$
\frac{\partial(\rho u \phi)}{\partial x}+\frac{\partial(\rho v \phi)}{\partial y}-\frac{\partial}{\partial x}\left[\Gamma_{\phi}\left(\frac{\partial \phi}{\partial x}\right)\right]-\frac{\partial}{\partial y}\left[\Gamma_{\phi}\left(\frac{\partial \phi}{\partial y}\right)\right]-S_{\phi}=0
$$

where $\phi$ stands for any of the variables to be solved, $\Gamma_{\Phi}$ is the diffusion coefficient, and $S_{\Phi}$ is the source term of the variable $\phi$. For $\phi=u$ or $v$ and $\Gamma_{\Phi}=\mu$ one gets the 
momentum equations, while for $\phi=1$ and $\Gamma_{\Phi}=0$ one obtains the continuity equation [21]. If $\phi=T$ and $\Gamma_{\Phi}=k_{t} / c_{p}$ one gets the energy equation. When $\phi=c$ and $\Gamma_{\Phi}=D$, the general equation stands for the mass transfer equation $[7,13]$.

Specification of control volume (CV) and the geometrical arrangement of the nine grid points to be used in the QUICK scheme are given in Fig. 2, in which the grid has been staggered so that the velocity grid points are located mid-way between the scalar grid points. The $\phi$ cells serve as grid points to store all scalar variables (pressure, temperature and concentration). All the governing equations are discretised by first integrating them over a $\mathrm{CV}$ and then approximating the fluxes of variable crossing every faces of each cell in term of the values at the neighboring grid points. In the present work, a QUICK scheme, which can handle uniform and non-uniform grid systems, is used to finite difference the convective terms and to secure second order accuracy in central differencing the diffusive fluxes. The resulting finite-difference equations are described in the form of [21]

$$
\begin{array}{ll}
a_{p} \phi_{p}=\sum_{i} a_{i} \phi_{i}+S_{u} \Delta V, & i=E, W, N, S, E E, W W, N N, S S, \\
a_{p}=\sum_{i} a_{i}-S_{p} \Delta V, & i=E, W, N, S, E E, W W, N N, S S,
\end{array}
$$

where $\Delta V$ is the cell volume and $S_{p}$ and $S_{u}$ are the coefficient appearing in the following linearized source term;

$$
S_{\phi}=S_{u}+S_{p} \phi
$$

The finite difference coefficients $a_{i}$ are the coefficients describing the magnitudes of the sum of the convective and diffusive fluxes and contain the geometric properties of the control volume. They are given in Abdel-Rahman's thesis [22].

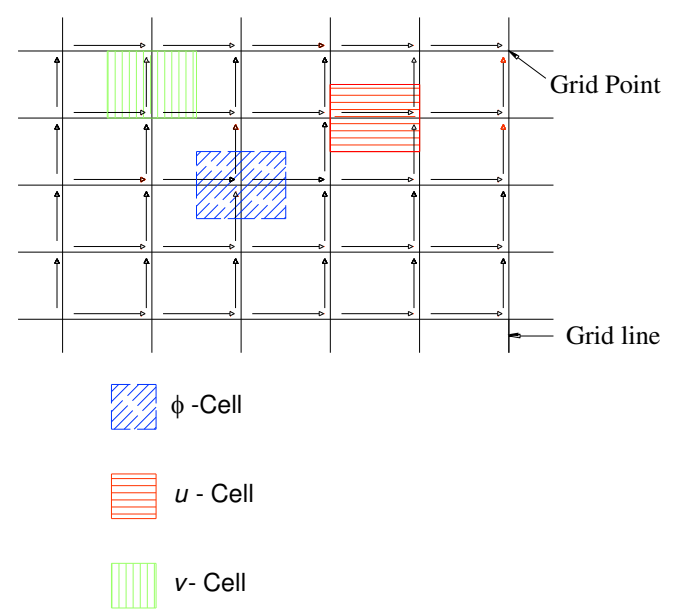

(a)
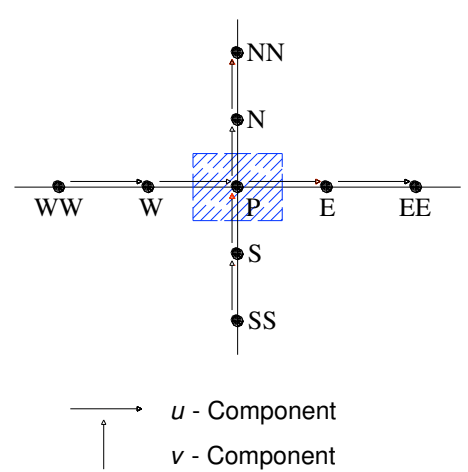

(b)

Figure 2: (a) Control volume specification and (b) Geometrical arrangement of the nine points used for the QUICK scheme [22]. 


\subsection{Boundary Conditions}

The boundary conditions of the problem are shown in Fig. 3.

1- At the inlet, the flow is assumed to be fully developed thus a parabolic flow is specified. A uniform inflow concentration of $c_{o}$ is specified. A constant inlet temperature $T_{o}$ is specified.

2- At the symmetry plane, $v=0$, and the normal gradients of the tangential velocity $u$, the concentration and temperature are set to zero.

3- At the membrane walls, the conditions are more complex, as flow permeates through the wall. For a membrane located at the plane $y=h$, a constant rejection and constant or variable permeation rate were specified. The tangential velocity $u$ is set to zero i.e. no slip at membrane wall, which characterizes the flows with solid bounding walls. Singh and Laurence [16], showed the existence of a slip-velocity at a porous surface on the basis of the theoretical and experimental work of Beavers and Joseph [23]. Accordingly, the slip has been taken into consideration in some cases during this study. Temperature at the membrane wall $T_{w}$ was set to a constant value. Variation in permeation was modeled using the following expression proposed by Brian [24] referenced by Wiley and Fletcher [8];

$$
v_{w}=v_{w o}\left[1-\beta\left(\frac{c_{w}}{c_{o}}-1\right)\right]
$$

The boundary condition on the concentration results from a balance of the convective and diffusive fluxes. In addition, it must be taken into account the fact that not all of the solute permeates through the membrane. This is done via the use of a rejection coefficient, $f$, and the concentration boundary condition is given by;

$$
D \frac{\partial c}{\partial y}+v_{w} f c_{w}=0
$$

4- At the exit, both the flow, temperature and concentration fields are assumed to obey the boundary layer approximation. It is important to mention that this treatment of the down stream end boundary condition has proved to be robust and effective in shortening the computational domain leading to the reduction of the number of grid nodes $[22,25]$.

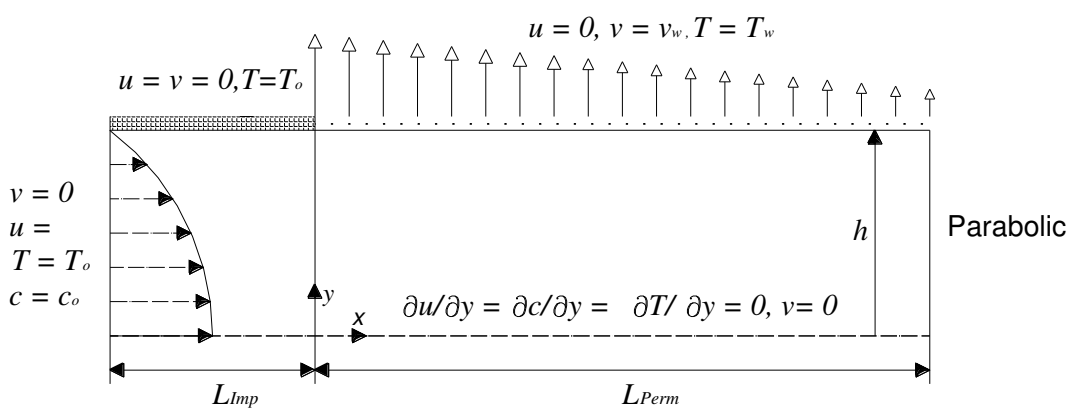

Figure 3: Boundary conditions. 


\subsection{Numerical procedure}

The present study utilizes a modified version of the SIMPLE procedures developed by Partaker and Spalding [21]. The main steps of the SIMPLE algorithm are;

1. A pressure field is assumed,

2. It is used to obtain approximate velocity field,

3. The velocity and pressure fields are corrected if the former does not satisfy the continuity equation,

4. Solve the discretization equations for the other $\phi$ 's such as temperature and concentration provided their influence on the flow field.

5. Return to step 2 with the corrected velocity field and the new values of all other $\phi$ 's and then the process 2-4 are repeated until a converged solution is obtained.

In the present work, the cross-stream distribution of $u$-velocity component is adjusted to satisfy the overall continuity (conservation of the mass flow are integrated over a cross-stream line) whereas the pressure field is adjusted to satisfy the overall momentum balance. This procedure is important especially for the present problem in which the flow is changed as the flow moves downstream due to the suction of the permeate flux from the membrane surface [22,25]. Moreover, the cross-stream distribution of the concentration is adjusted to satisfy the overall mass concentration of the permeated species.

An alternating direction implicit (ADI) procedure, which has been developed by Abdel-Rahman and Suzuki [25], has been combined with the iterative solution procedure of equations (7) to enhance isotropic propagation of a change of variables occurring at one point to the surrounding. This procedure makes use of the line-by-line TDMA solver. In the ADI procedure, sweep of line- by-line integration was carried out along both north-south grid lines and along east-west grid lines alternatively. The same procedure was applied twice for the pressure correction [22].

\subsection{Physical Properties}

For validation of the present model, the physical properties of solution were taken as the same of those used by the other researches. However, in the last section of this study the solvent was a pure sucrose solution with a concentration corresponding to the raw cane juice. Expressions for the variation of physical properties with temperature and concentration for sugar solution were taken from sugar technologist manual edited by Bubnik et al . [26].

\section{RESULTS AND DISCUSSION}

On the light of the present objective of this work, the validity of the present numerical simulation of flow in membrane modules will be verified by comparing results of this model with the existing analytical method by Berman [20] and Singh and Laurence [16]. The differences in these models are that the slip velocity at the membrane wall was neglected in the Berman's solution, while it was taken into consideration by Singh and Laurence's model. Moreover, various features of the numerical simulation will be demonstrated. 


\subsection{Flow model Validation}

The results obtained at different axial position of $x / h$ for $R e_{o}=10^{3}$ and $R e_{w}= \pm 1$ along with the Berman [20] solution are shown in Figs. 4 and $\mathbf{5}$. Figures 4 and $\mathbf{5}$ show that the values of $u$ component along the length of channel obtained by the present model agree very well with Berman solutions for both suction $\left(R e_{w}=1\right)$ and injection $\left(R e_{w}=-1\right)$ wall velocity.

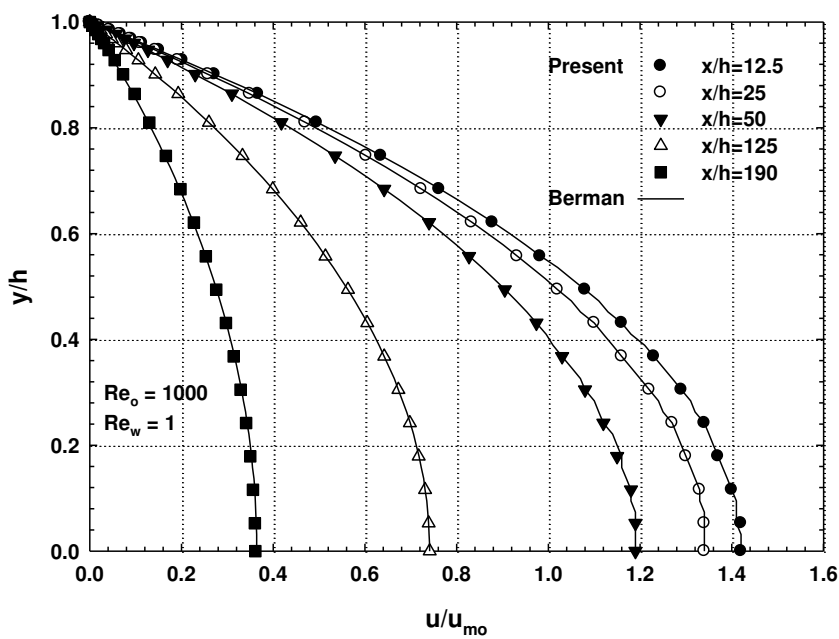

Figure 4: Comparison of the streamwise velocity profile at different $x / h$ with the solutions of Berman [20] in case of wall suction.

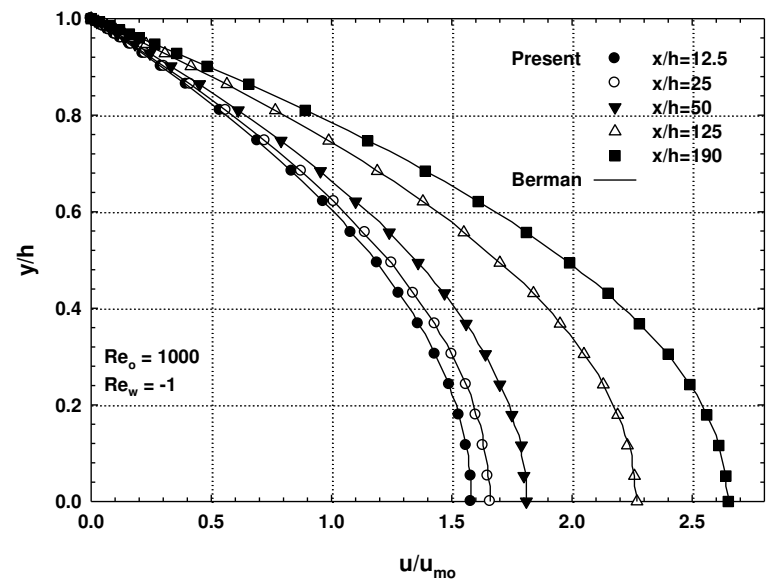

Figure 5: Comparison of the streamwise velocity profile at different $x / h$ with the solutions of Berman [20] in case of wall injection.

The effect of slip coefficient $\phi_{s}$ on the velocity profiles is also validated by comparing the present model with the solutions of Singh and Laurence [16] as shown in Fig. 6 for a main flow Reynolds number of 1000 and a longitudinal $x / h$ position equal to 70 . The curves are plotted for slip coefficient, $\phi_{s}$ equal to $0,0.1$ and 0.5 , and a 
wall Reynolds number, $R e_{w}$ of 0.1 . Figure 6 shows that the velocity at the membrane surface $(y / h=1)$ is 0 , when $\left(\phi_{s}\right.$ is 0$)$ as expected. As the slip velocity increases with increasing $\phi_{s}$, the wall shear decreases and the profiles become flatter. The present model shows an excellent agreement with the solutions of Singh and Laurence [16]. Results shown in Figs. 4, 5, and $\mathbf{6}$ prove the ability of the present model to predict the hydrodynamic behaviors of the laminar channel flow with injection or suction in both cases of flow with or without slip. The next step is to use the present model for the prediction of the $\mathrm{CP}$.

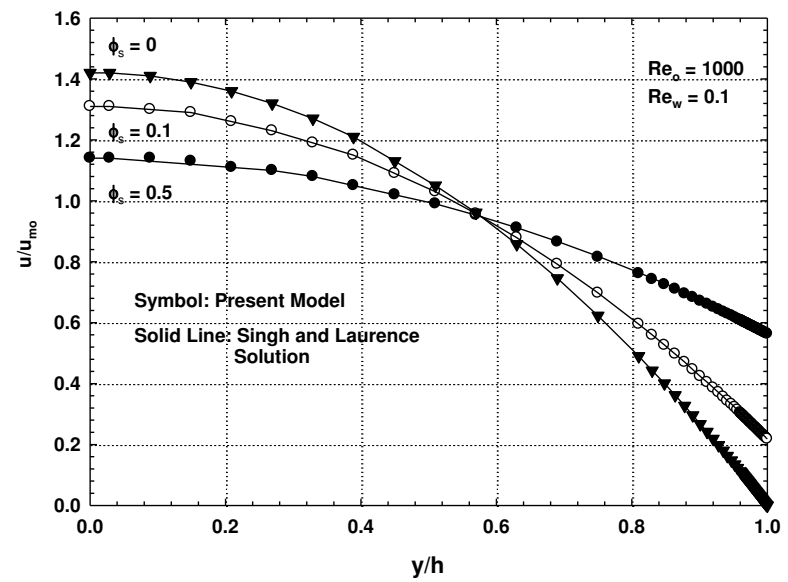

Figure 6: Comparison of the streamwise velocity profile at different slip coefficients with the solutions of Singh and Laurence [16].

\subsection{Prediction of Concentration Polarization}

\subsubsection{Constant permeation and constant properties}

For the numerical model under consideration, the effect of the main flow Reynolds number, the wall Reynolds number and the diffusivity coefficient on the concentration polarization and flow field will be examined. The following was assumed:

1. The properties are constants.

2. The rejection coefficient was set to 1 .

3. The permeation velocity was set constant along the channel.

4. The slip coefficient was set to zero.

5. The inlet concentration was $2 \times 10^{-3}(\mathrm{Kg} / \mathrm{Kg})$.

\subsubsection{Effect of main flow Reynolds number}

Three different flow rates with main flow Reynolds number of 250, 500 and 1000 were considered to explore its effect on the model characteristics. The wall Reynolds number was 0.1 and the $S c$ number for all cases was 100 . Figure 7 shows the axial velocity profile across the channel at the distance of $x / h=70$ along with their counterparts obtained from Berman [20] solutions. 
Figure 7 shows that the increase in the main flow Reynolds number causes an increase of the $u$ velocity that is physically acceptable. It also shows an excellent agreement between the present model and Berman solutions.

Figure 8 shows that the solute concentration at the membrane surface is decreased when the main flow Reynolds number is increased. The results shown in this figure agree with physical facts as the concentration near the membrane surface becomes smaller when the main flow Reynolds number is increased.

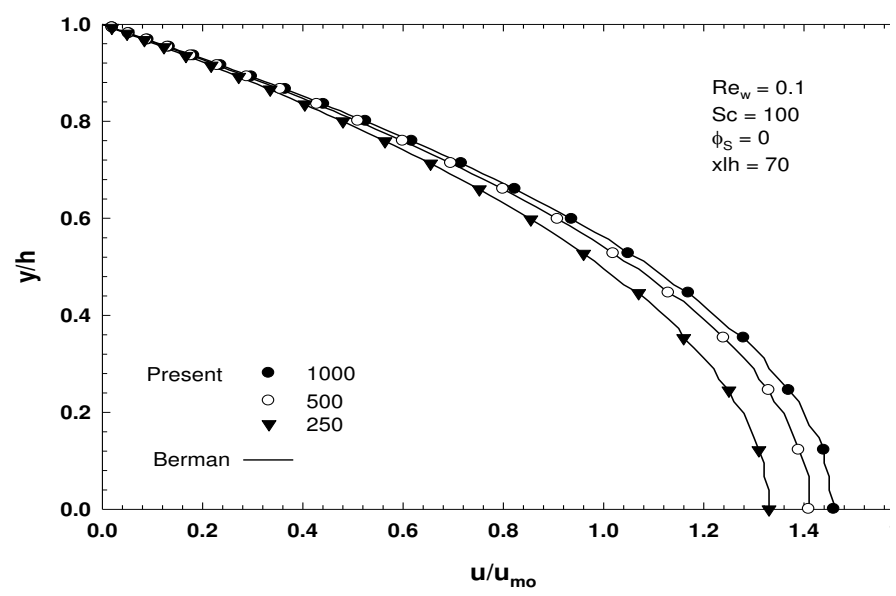

Figure 7: Streamwise velocity profile at $x / h=70$ as a function of $R e_{o}$.

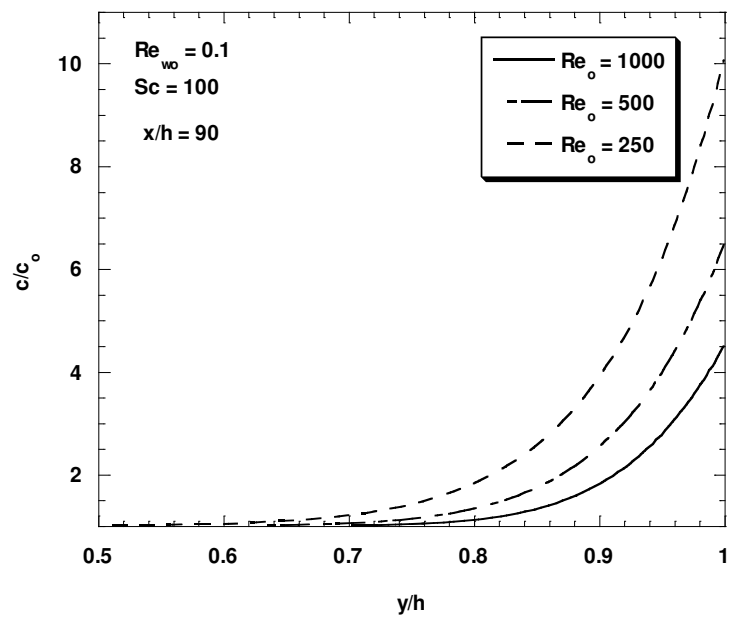

Figure 8: Effect of $R e_{o}$ on concentration profile at $x / h=90$.

Figure 9 shows the variation of the local concentration boundary layer thickness along the membrane length for different main flow Reynolds number. Here, the concentration boundary layer is defined as the distance from the membrane surface to 
the location where $\left(c-c_{o}\right) / c_{o}$ is greater or equal to $10^{-3}$, It is clear from the figure that high main flow Reynolds number impedes the accumulation of solutes on the membrane surface.

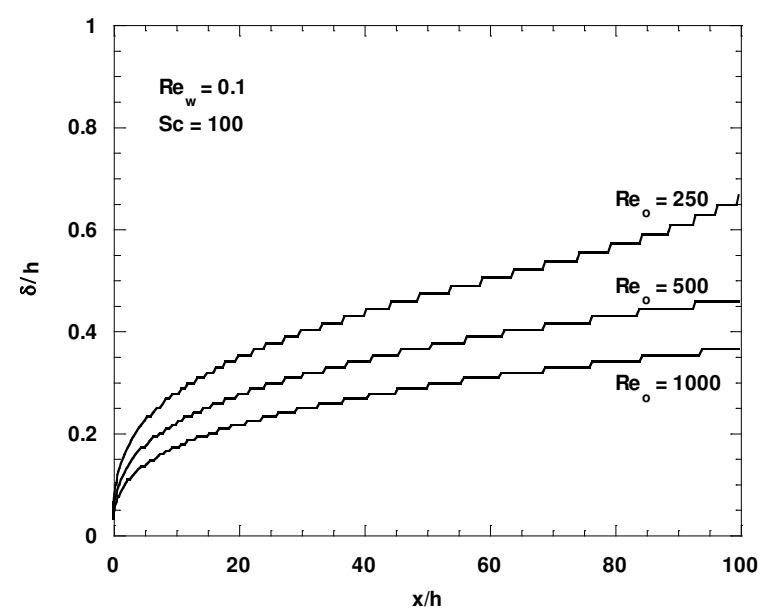

Figure 9 : Effect of $R e_{o}$ on the local thickness of concentration boundary layer along the membrane length.

\subsubsection{Effect of wall Reynolds number}

Three permeate velocities corresponding to wall Reynolds number of $0.02,0.1$ and 0.2 were studied to examine the effects of changing the $R e_{w}$ on the model behavior. The main stream Reynolds number was 1000 and the $S c$ number was set to 100 .

More particles are expected to be convectively driven to the membrane surface as the permeate velocity is increased as shown in Fig. 10. When the wall Reynolds number increases due to an increase in transmembrane pressure, the concentration at the membrane surface is increased, because more particles are driven to the membrane surface. These numerical results are consistent with the results of Lee and Clark [12] and Damak et al. [15].

\subsubsection{Effect of diffusivity coefficient}

A study to explore the effects of the diffusivity coefficient on the model behavior using diffusivity coefficients corresponding to $\alpha_{D}=0.1,0.2,0.5$, and 1.0 was considered. The main flow and wall Reynolds numbers were set constant at 1000 and 0.2 , respectively. The effect of $\alpha_{D}$ on the concentration polarization is shown in Fig. 11 . These results are plotted in a manner suggested by Singh and Laurence [16]. Where, $\xi$ is a non-dimensional parameter represents the water recovery factor and $C p$ is the concentration polarization given by Geraldes et al. [7];

$$
\xi=\frac{v_{w o}}{u_{m o}} \cdot \frac{x}{h}, \quad \text { and } \quad C p=\frac{c_{w}(1-\xi)}{c_{o}}-1
$$




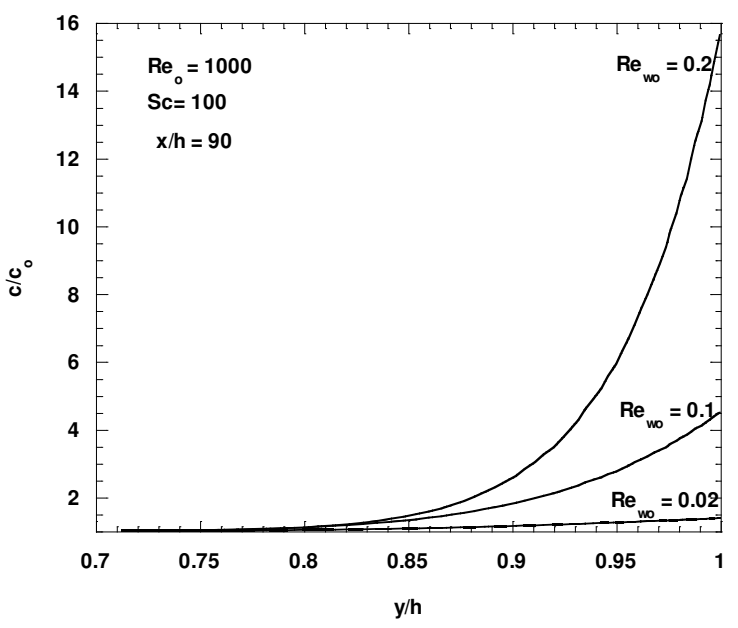

Figure 10: Effect $R e_{w o}$ on the concentration profile at $x / h=90$.

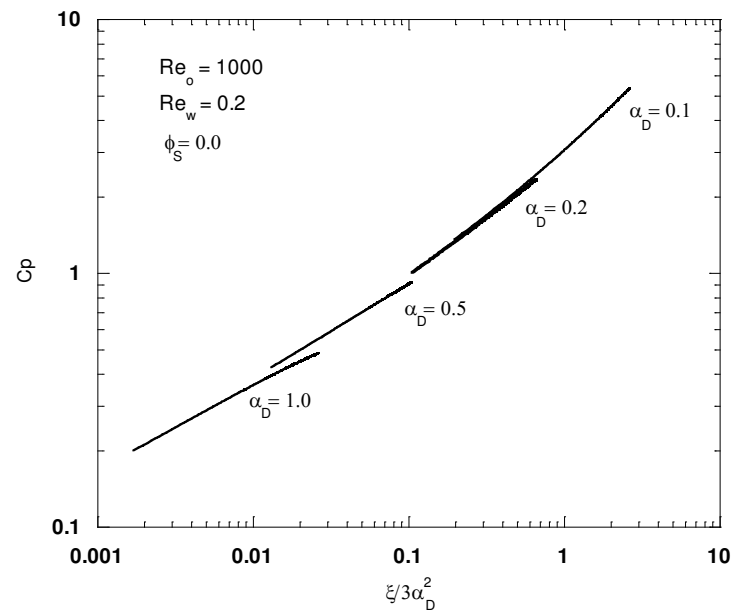

Figure 11: Concentration polarizations as a function of longitudinal position from the membrane entrance.

Figure 11 indicates that at $\alpha_{D}=0.1$ the concentration polarization has the highest value. As the value of $\alpha_{D}$ increases, the concentration polarization is decreased. Actually, an increase in the value of $\alpha_{D}$ represents an increase in the value of the diffusion coefficient $D$, which leads to an increase in back-diffusion of solute from the membrane surface for the same value of $v_{w}$ and $h$. Therefore, the concentration polarization is decreased with increasing the $\alpha_{D}$. Figure 11 also shows that for any value of $\alpha_{D}, C_{p}$ is increased with the longitudinal position from the membrane entrance.

In a similar manner, Fig. 12 shows the concentration profiles at various axial locations from the membrane entrance. The concentration near the membrane surface increases with the axial distance from the channel inlet, which means that the thickness of concentration boundary layer increases also along the membrane from the inlet to the outlet. This finding is consistent with that classically found by many authors $[13,15]$. 


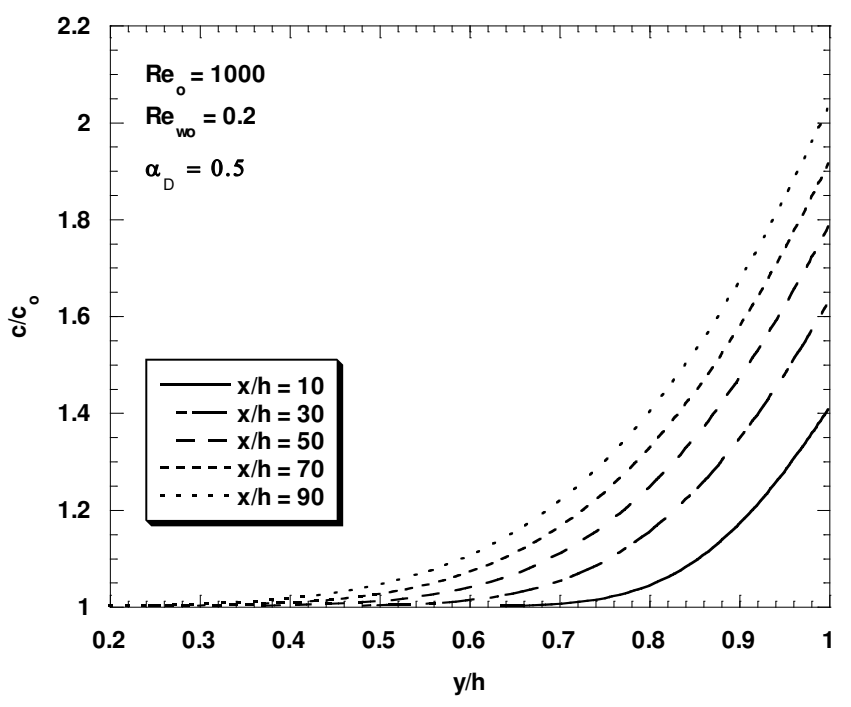

Figure 12: Concentration profiles at different $x / h$.

\subsubsection{Variable wall permeation and constant properties}

\subsubsection{Effect of rejection}

It is clear from Figs. $\mathbf{1 1}$ and $\mathbf{1 2}$ that the concentration at the membrane wall is increased along the membrane length. Therefore, it is necessary to reflect the effect of variable wall concentration on the wall permeation. Thus, the wall permeation velocity $v_{w}$ was set as a function of concentration according to Eq. (8).

Four numerical experiments were carried out to compare the results obtained for variable wall flux ( $\beta=0.25, \alpha_{D}=0.27$ ) with those obtained for constant wall flux at constant physical properties and constant rejection of 1.0 or 0.9 . The main flow Reynolds number and the wall Reynolds number were set to 1000 and 0.0625 , respectively. The feed concentration was set to $2 \times 10^{-3}(\mathrm{Kg} / \mathrm{Kg})$. These results are shown in Fig. 13.

Figure 13 shows that there is almost no effect of wall flux variations on the wall concentration as long as the rejection is constant. On the other hand, Fig. 13 shows that the change in rejection from 1 to 0.9 has small effects on the wall concentration especially in the downstream end of the membrane. This is consistent with the results of Wiley and Fletcher [8]. This could be explained partially due to the small axial variation of the wall velocity as $\beta=0.25$. However, the concentration polarisation increases along the membrane channel, as it is clear from the figure.

Figure 14 compares the variable wall velocity along the channel length at different rejection. As is shown in the figure, at fixed length from the membrane entrance, the wall velocity at the higher rejection value is smaller than its counterpart at the smaller rejection value. This is normal as the wall velocity is set as a function of the concentration, which increases with rejection as is shown in Fig. 13. 


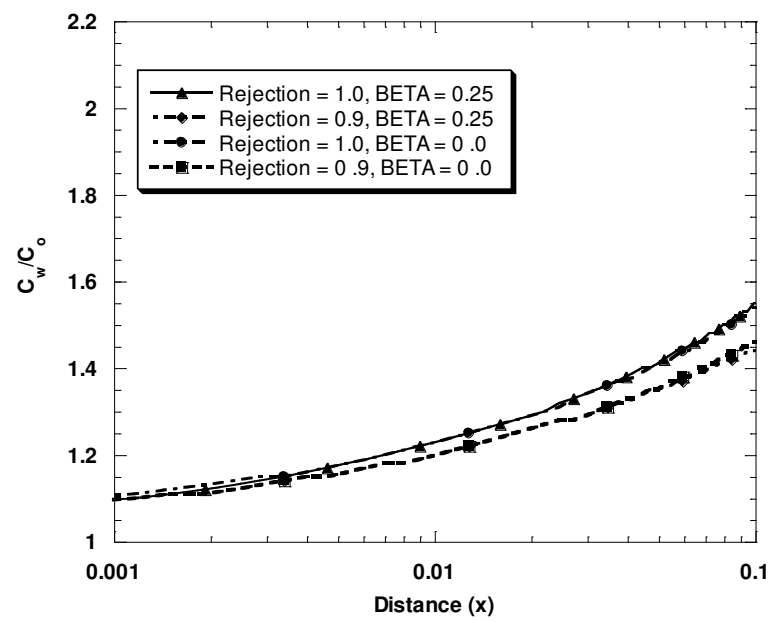

Figure 13: Effect of variable wall flux $(\beta=0.25)$ and rejection on the wall concentration.

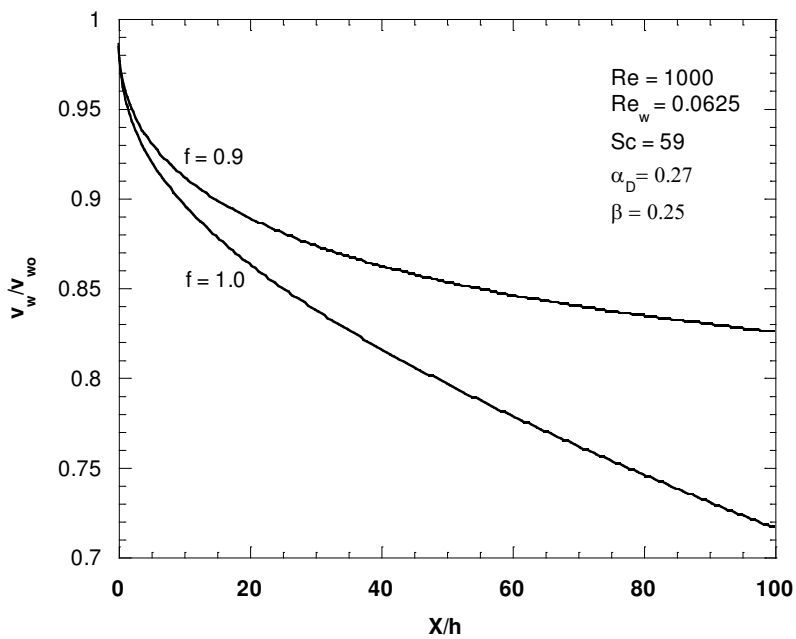

Figure 14: Effect of rejection on the variable wall velocity $(\beta=0.25)$ along the membrane length.

\subsubsection{Effect of slip coefficient}

The Effect of slip coefficient in case of variable wall velocity $\left(\beta=0.25, \alpha_{D}=\right.$ $0.27, f=0.9$ ) on the flow and concentration profiles is also studied. Figure 15 shows the effect of slip coefficient on streamwise velocity for constant and variable wall velocity. The figure shows that the velocity at the membrane surface $(y / h=1)$ is 0 , in case of no slip $\left(\phi_{s}=0\right)$ as expected. As the slip velocity is set to predetermined value $\left(\phi_{s}=0.5\right)$, the wall shear decreases and the profiles become flatter as in the case of constant wall velocity. In addition, there is no effect of the wall velocity variation on the streamwise velocity in case of no $\operatorname{slip}\left(\phi_{s}=0\right)$. 
The effect of an increase in $\phi_{s}$ is to decrease the value of the wall concentration and the local thickness of the concentration polarization layer as shown in Figs. 16 and 17.

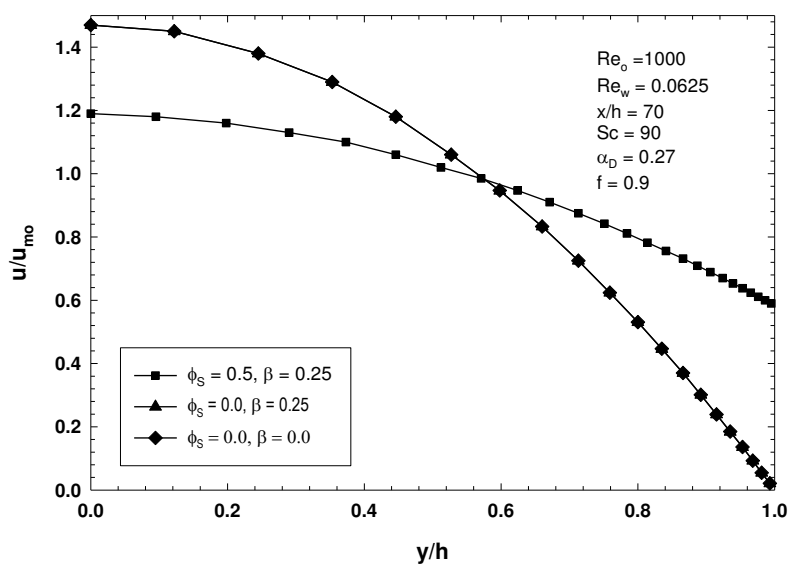

Figure 15: Velocity profile at different slip coefficients in case of variable wall flux $\left(\beta=0.25, \alpha_{D}=0.27\right)$ at $x / h=70$.

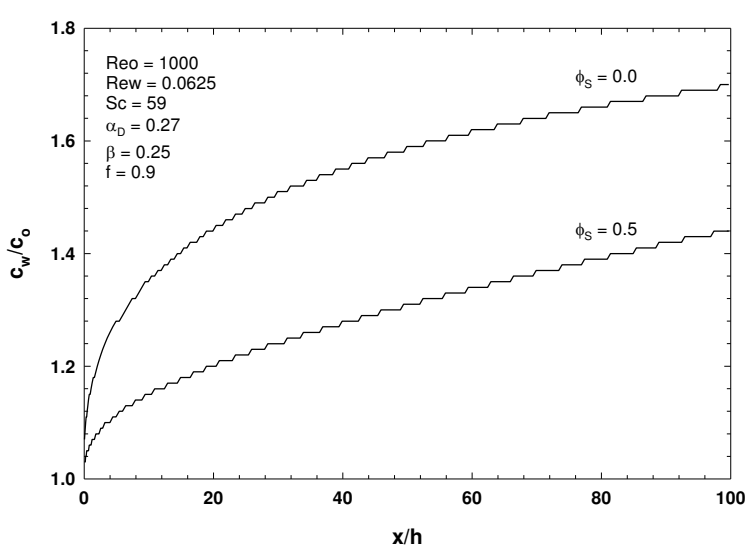

Figure 16: Effect of slip coefficient on the wall concentration along the membrane length in case of variable permeate velocity $(\beta=0.25)$.

\subsubsection{Variable wall permeation and variable properties}

\subsubsection{Effect of slip and rejection}

Numerical experiments were carried out on conditions similar to those of the experimental work of Abbara et al. [27]. The effects of different permeation flux, 
rejection and inlet temperature were examined. This solution was a pure sucrose with concentration of $0.13(\mathrm{~kg} / \mathrm{kg})$. In all cases, the mainstream Reynolds number was set to a constant value of 1700. The wall Reynolds number was set to 0.02 and 0.05 . Rejection was set to 0.1 and 0.2 . The inlet temperature of the solution was set to 50 and $70{ }^{\circ} \mathrm{C}$. The temperature of sugar solution enters the channel is higher than the temperature of the membrane wall. Thus, the energy equation was also solved. Change in density, viscosity, specific heat, thermal conductivity and diffusion coefficient as a function of both temperature and concentration was also considered.

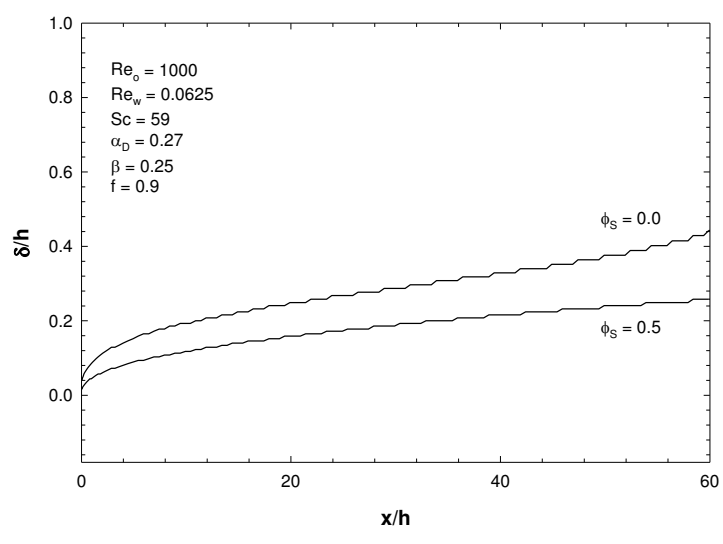

Figure 17: Effect of slip coefficient on the local thickness of the concentration boundary layer in case of variable permeate velocity $(\beta=0.25)$.

Figure 18 shows the effect of wall Reynolds number and rejection of the sugar solution on the concentration profile at $x / h=700$. It is clear from the figure that at the same wall Reynolds number, the wall concentration increases with the increasing rejection from 0.1 to 0.2 . This is attributed to the accumulation of more sugar molecules at the membrane surface at the higher value of rejection. Moreover, Fig. 18 shows that at the same value of rejection, increasing the wall Reynolds number from 0.02 to 0.05 decreases the concentration up to certain value of $y / h$ (depends on the value of rejection $f$ ) before it recovers near the wall. However, the increase in wall concentration is not significant.

\subsubsection{Effect of temperature}

Effect of variation of both the temperature and rejection on the concentration profile and the wall permeation velocity along the membrane channel is shown in Figs. 19 and 20. These numerical computations were carried out to explore the effects of changing the physical properties especially, the diffusivity coefficient and the viscosity with temperature and concentration. Figure 19 shows that at the same temperature the wall concentration increases with increasing the rejection from 0.1 to 0.2 . Figure 19 shows also that the effect of temperature on the wall concentration is negligible at the lower rejection value of 0.1 . However, the rise of temperature from 50 
${ }^{\circ} \mathrm{C}$ to $70{ }^{\circ} \mathrm{C}$ decreases the wall concentration slightly but increases the concentration noticeably everywhere in the channel for both rejection values.

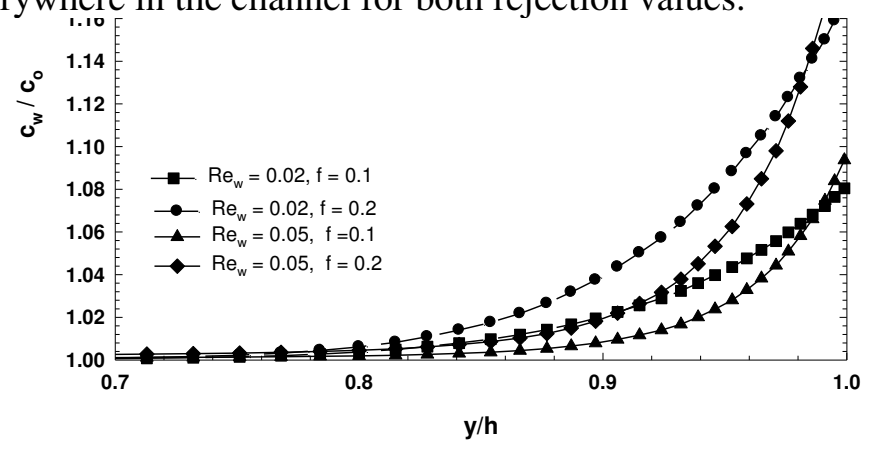

Figure 18: Effect of variable wall flux $(\beta=0.25)$ and rejection on the concentration

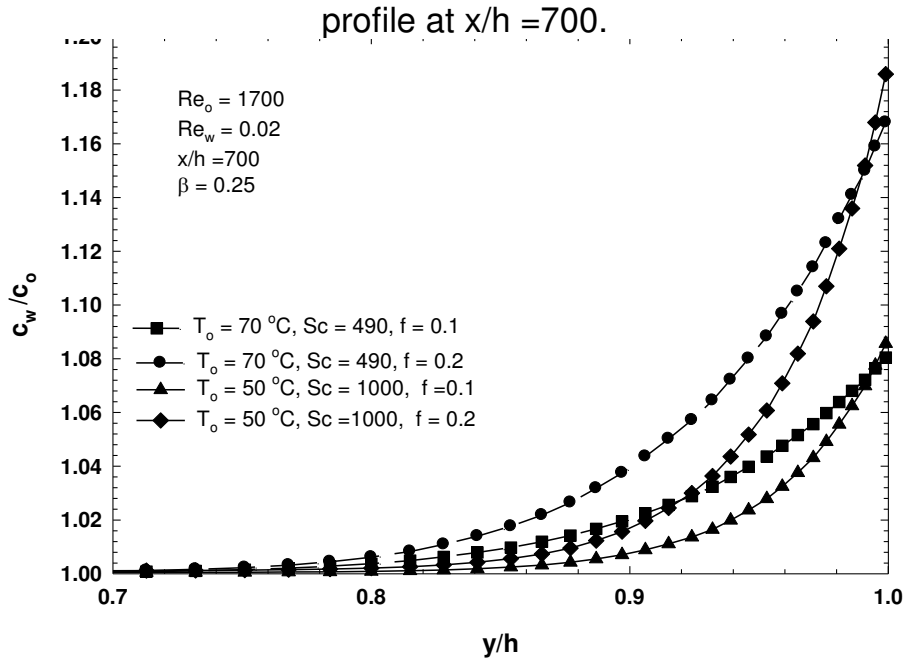

Figure 19: Effect of temperature and rejection on the concentration profile at $x / h=700$.

As it is expected, Fig. $\mathbf{2 0}$ shows that the effect of increasing the temperature is to increase the wall velocity for the higher rejection value of 0.2 . This could explain the behavior of the concentration profile shown in Fig. 19. 


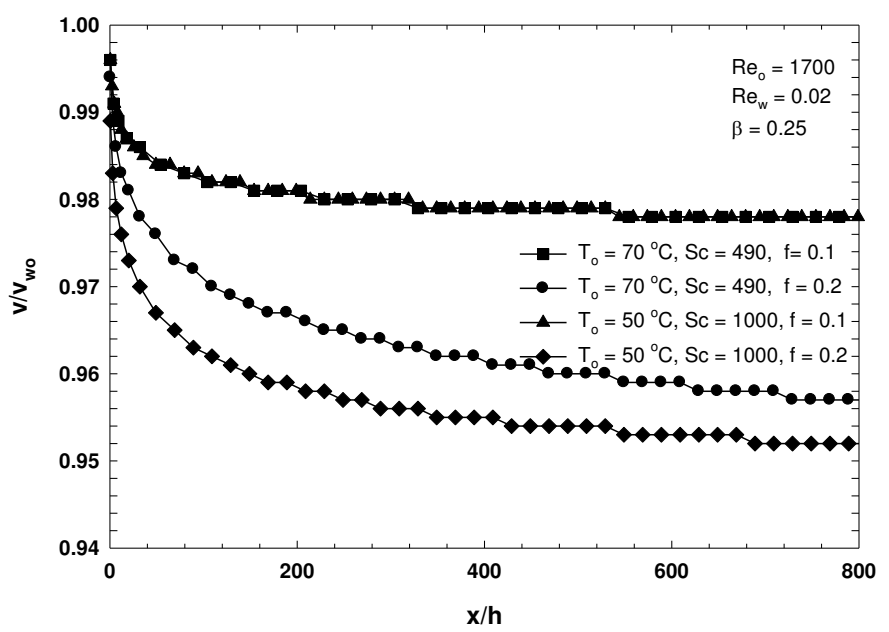

Figure 20: Effect of temperature and rejection on the wall velocity along the membrane length.

\section{CONCLUSIONS}

Laminar flow and related mass transfer in channels representing the membrane module has been numerically investigated. This was facilitated by solving the elliptic type of the governing equations using the SIMPLE pressure-correction algorithm for pressure field in connection with the high order QUICK scheme. The alternating direction implicit ADI scheme, which makes use of the TDMA in solving the resulting coefficient matrix, was used to solve the governing equations to reduce the number of iterations. A scheme to secure the overall mass conservation was also employed. The following points can be drawn from the numerical simulation:

- Results of numerical simulation of the mass transfer in membranes agree very well with the previous studies.

- The numerical study highlights the necessity of the fine mesh grid near the membrane wall to cop with the very steep changes in concentration there, especially for the case of small diffusion coefficient.

- As the solute concentration is not constant at the membrane wall, the numerical study pointed to the importance of making the permeate velocity varying with the concentration.

- The numerical solutions suggested that the effects of temperature were insignificant in case of low rejection of pure sucrose solution. Temperature effects were rather noticeable for the high rejection.

The simulation model developed in this work can be directly applied to a number of industrial membrane processes. The code developed here was designed so 
that any model describing the dependence of permeates flux and solute rejection on process parameters can easily be introduced into the simulation program. The models for modules other than channel could also be handled by this code.

\section{REFERENCES}

[1] Miranda, J. M. and Campos, J. B. L. M.(2001), Concentration polarization in a membrane placed under an impinging get confined by a conical wall- a numerical approach. Journal of Membrane Science, Vol. 182, pp. 257-270.

[2] Barros, S. T. D. de, Andrade, C. M. G., Mendes, E. S. and Peres, L. (2003), Study of fouling mechanism in pineapple juice clarification by ultrafiltration. Journal of Membrane Science, Vol. 215, pp. 213-224.

[3] Hong, S., Faibish, R. S. and Elimelech, M. (1997), Kinetics of permeate flux decline in crossflow membrane filtration of colloidal suspensions. Journal of Colloid and Interface Science, Vol. 196, pp. 267- 277.

[4] Richardson, C. J., Nassehi, V. (2003), Finite element modelling of concentration profiles in flow domains with curved porous boundaries. Chemical Engineering Science, Vol. 58, pp. 2491 - 2503.

[5] Paris, J., Guichardon, P. and Charbit, F.(2002), Transport phenomena in ultrafiltration: a new two-dimensional model compared with classical models. Journal of Membrane Science, Vol. 207, pp. 43-58.

[6] Kovasin, K. (2002), Modeling ultrafiltration and filtration phenomena applied in chemical pulping processes, Chemical Engineering Report Series, Espoo.

[7] Geraldes, V., Semião, V. and Pinho, M. N. de (2000), Numerical modelling of mass transfer in slits with semi-permeable membrane walls, Engineering Computations, Vol. 17, No. 3, pp. 192-217.

[8] Wiley, D. E. and Fletcher, D. F. (2003), Techniques for computational fluid dynamics modeling of flow in membrane channels, Journal of Membrane Science, Vol. 211, pp. 127-137.

[9] Dendukuri, D., Karode, S. K. and A. Kumar (2005), Flow visualization through spacer filled channels by computational fluid dynamics-II: improved feed spacer designs, Journal of Membrane Science, Vol. 249, pp. 41-49.

[10] Elimelech, M. and Bhattacharjee, S. (1998), A novel approach for modeling concentration polarization in crossflow membrane filtration based on the equivalence of osmotic pressure model and filtration theory, Journal of Membrane Science, Vol. 145, pp. 223 - 241.

[11] Huang, L. and Morrissey, M. T. (1998). Fouling of membranes during microfiltration of surimi wash water: Roles of pore blocking and surface cake formation, Journal of Membrane. Science, Vol. 144, pp. 113-123.

[12] Lee, Y. and Clark, M. M. (1997), A numerical model of steady-state permeate flux during cross flow ultrafiltration, Desalination, Vol.109, pp. 241.

[13] Geraldes, V., Semião, V. and Pinho, M.N.de (2001), Flow and mass transfer modelling of nanofiltration, Journal of Membrane Science, Vol. 191, pp.109128.

[14] Damak, K., Ayadi, A., Schmitz, P. and Zeghmati, B. (2004), A new NavierStokes and Darcy's law combined model for fluid flow in crossflow filtration tubular membranes, Desalination, Vol. 161, pp.67-76. 
[15] Damak, K., Ayadi, A., Schmitz, P. and Zeghmati, B. (2004), Modeling of crossflow membrane separation processes under laminar flow conditions in tubular membrane, Desalination, Vol. 168, pp. 231-239.

[16] Singh, R. and Laurence, R. L. (1979), Influence of slip velocity at a membrane surface on ultrafiltration performance $-\mathrm{I}$. Channel flow system, International Journal of Heat and Mass Transfer, Vol. 22, pp. 721-729.

[17] Yeh, H. M. and Cheng, T. W. (1999), Analysis of the slip effect on the permeate flux in membrane ultrafiltration, Journal of Membrane Science, Vol. 154, pp. 41-51.

[18] Anderson, D.A., Tannehill, J.C. and Pletcher, R.H. (1984), Computational fluid mechanics and heat transfer, Hemisphere Publishing Corporation, NewYork.

[19] Huang, L. and Morrissey, M. T. (1999), Finite element analysis as a tool for crossflow membrane filter simulation, Journal of Membrane Science, vol. 155, pp. 19-30.

[20] Berman, A. S. (1953), Laminar flow in channels with porous walls, Journal of Applied Physics, Vol. 24, pp. 1232-1235.

[21] Partakar, S. V. and Spalding, D. B. (1972), A calculation procedure for heat, mass and momentum transfer in three-dimensional parabolic flows, International Journal of Mass and Heat Transfer, Vol. 15, pp. 1787-1806.

[22] Abdel-Rahman, A. K. (1992), Flow and heat transfer characteristics of internal flows with fluid injection, PhD thesis, Kyoto University, Kyoto, Japan.

[23] Beavers, G.S. and Joseph, D.D. (1967), Boundary conditions at a naturally permeable wall, J. Fluid Mech., Vol. 30, pp. 197-207.

[24] Brian, P.L.T. (1965), Concentration polarization in reverse osmosis desalination with variable flux and incomplete salt rejection, Ind. Eng. Chem. Fund., Vol. 4, pp. 439-445.

[25] Abdel-Rahman, A. K. and Suzuki, K. (1995), Laminar channel flow with fluid injection accounting for the flow in the porous wall, Proceedings of the 5th Int. Conference of Fluid Mechanics, Cairo, pp. 367-379.

[26] Bubnic, Z., Kadlec, P., Urban, D. and Bruhns, M. (1995), Sugar technologists' manual-chemical and physical data for sugar manufactures and users, Bartens, Berlin, Germany.

[27] Abbara, A. A., Ali K. Abdel-Rahman, and M.R. Bayoumi (2004), Microfiltration and ultrafiltration of sugarcane juice in the production of plantation white sugar, Proceedings of STCEX, Saudi Arabia, Ryadh, pp. 151158. 


\section{النمذجة العددية للتركيز بداخل القنوات ذات الاغشية}

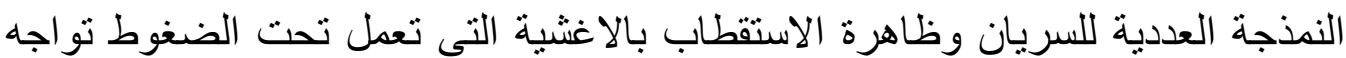

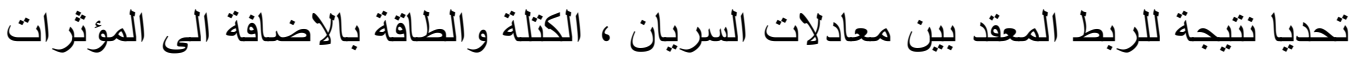

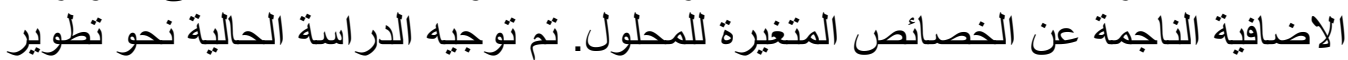

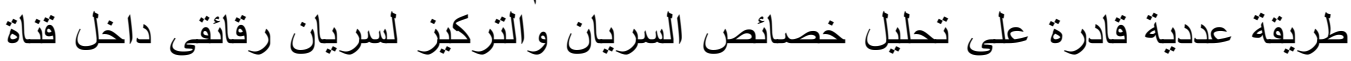

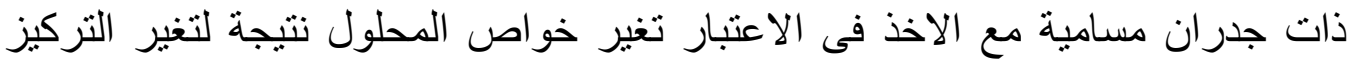

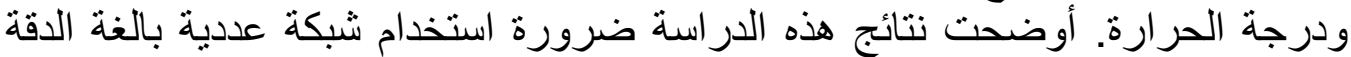

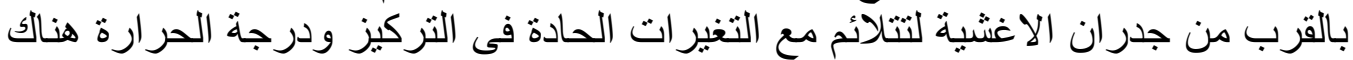

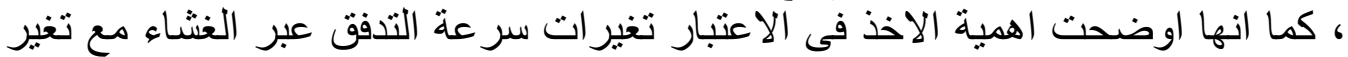

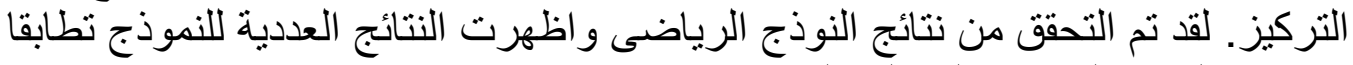
جيدا مع النتائج العددية و المعملية السابقة. 